\title{
Awareness of Oral Corticosteroid Use among Sudanese with Rheumatological Diseases: A Single Centre Study
}

Waleed Azhary Sir Alkhatim ${ }^{1}$, Yousif Abdallah Adam ${ }^{1 *}$, Esraa M Attaalmanan ${ }^{1}$, Samah A M Idriss ${ }^{1}$, Alzahra A Mohamed Baiker ${ }^{3}$, Ziryab Imad Taha ${ }^{1}{ }^{2}$

\author{
${ }^{1}$ Sudan medical specialization Board - Khartoum, Sudan \\ ${ }^{2}$ Department of Internal Medicine, Faculty of Medicine, University of Bahri, Khartoum, Sudan \\ ${ }^{3}$ University of Medical Sciences and Technology, Khartoum, Sudan
}

DOI: $10.36347 /$ sajp.2021.v10i03.003

| Received: 02.03.2021 | Accepted: 20.03.2021 | Published: 28.03.2021

*Corresponding author: Dr. Yousif Abdallah Adam

\section{Abstract}

Background: Corticosteroids (CS) have long been known as the most powerful of all known anti-inflammatory agents. They have shown pivotal role in the treatment of various serious disorders such as autoimmune diseases, allergic processes, organ transplantation, rheumatologic, pulmonary, hematological, ophthalmologic, gastrointestinal disorders, and others. Nevertheless, such massive clinical use does not come without risks, where prolonged use has been linked to several serious side effects. Therefore, careful consideration of precautions recommended for safe use of corticosteroids is of paramount importance. Aims and Objectives: To assess awareness in patients with rheumatological diseases toward oral corticosteroids, to explore the extent to which steroid outpatients were well engaged with medication proper use and safety-related behaviors. Materials and Methods: A structured questionnaire methodology was adopted to assess the awareness revolving around steroid usage in patients. The study was a total coverage of patients attending outpatient clinic of Omdurman military hospital. Data were collected by well-trained researchers, analyzed. Standard descriptive and analytical statistics were used to analyze the data. Results: Overall, our study showed poor awareness score (53\%) results among Sudanese patients using corticosteroids, but there was significant relation between awareness score and educational level. Conclusion: Despite the massive use of corticosteroids and its inherent associated risks, patients still needs provisions for better education regarding their medication efficacy, safety, and proper use. By careful follow-up of proper corticosteroids use instructions, both patients and physicians would positively reach an optimized cost-effective therapeutic approach with much more improved benefit/risk ratio.

Keywords: Corticosteroids (CS), anti-inflammatory agents, organ transplantation, ophthalmologic, rheumatological diseases.

Copyright $(\mathcal{C}) 2021$ The Author(s): This is an open-access article distributed under the terms of the Creative Commons Attribution 4.0 International License (CC BY-NC 4.0) which permits unrestricted use, distribution, and reproduction in any medium for non-commercial use provided the original author and source are credited.

\section{INTRODUCTION}

Since their discovery in the 1940s, Corticosteroids (CS) have become one of the most widely used and effective treatments for various inflammatory and autoimmune disorder [1]. Over the years it has become increasingly apparent that corticosteroids are being abused by doctors \& patients alike [2]. The side effects and complications of steroids are high among patient on long-term oral CS, because they do not receive adequate information's and basic monitoring before starting steroids from prescriber.

However, for most GC-related AEs, "threshold" dose or treatment duration has not been established [3]. Glucocorticoids (GC) -associated toxicity appears to be related to both the average dose and cumulative duration of GC use [4].

As research on corticosteroid therapy progressed in the $1980 \mathrm{~s}$, several studies popularized treatment using lower-dose corticosteroids $(<15 \mathrm{mg} / \mathrm{d})$, including a double-blind study that found that patients' conditions improved, with minimal AEs. Use of even lower doses of corticosteroids $(<5 \mathrm{mg} / \mathrm{d})$ gained recognition by primary care providers and rheumatologists as both a "bridge" and an alternative to slow-acting antirheumatic drugs, disease-modifying antirheumatic drugs, and NSAIDs [5]. 
Waleed Azhary Sir Alkhatim et al., Sch Acad J Pharm, Mar, 2021; 10(3): 49-53

Well-known adverse events (AEs) associated with systemic CS use include osteoporosis, cardiovascular disease, impaired immune response and wound healing, alterations in glucose and lipid metabolism, psychiatric disturbances, cataract, nausea/vomiting/ other gastrointestinal conditions, sleep disturbance, and hypertension [6, 7]. Such complications may decrease a patient's quality of life [8, 9].

Keeping this in view, several international guidelines have been set to help streamline CS use [10]. Basic knowledge about CS usage guidelines and adverse reactions appears to be imperative and should be highly stressed to all CS users.

\section{MATERIALS AND METHOD}

This is a prospective, descriptive, cross sectional hospital based study to assess the awareness in patients with rheumatologic diseases toward oral corticosteroids.

Also to estimate the patients knowledge, practice and behavior towards oral corticosteroids use.

\section{Subjects and Settings}

Total coverage of patients attending to rheumatology outpatient clinic of Omdurman Military hospital (Because there is no recent data or studies about prevalence or frequency of patient with rheumatological diseases in Sudan), in a period from April 2019 to August 2019. All adult patients with rheumatological diseases taking oral corticosteroids were chosen and others were excluded.

\section{Data Collection}

Data were collected using a well prepared questionnaire and well trained researchers. Participants were invited to complete the questionnaires by the researcher. ethical clearance was obtained from the ethical committee at Sudan Medical Specialization Board, as well as Omdurman military hospital administration approval.

\section{Data Variables}

Age, sex , residence, occupation, educational level, marital status, oral CS use side effects and complications (hypertension, hyperglycemia, osteoporosis, poor wound healing), main source of oral CS knowledge (health-care professional, media, websites, family and friends), drug-drug interactions, mood of stopping oral CS, taking supplements with oral CS.

\section{Statistical Analysis}

For analysis of data, Statistical Package for Social Sciences software, version 21.0 (IBM SPSS Inc., Chicago, IL) and STATA 11 were used. Initially, all information gathered via questionnaire then coded into variables. Normality of data was tested using
Kolmogorov-Smirnov test and Shapiro-Wilk test. Both descriptive and inferential statistics involving fisher's exact test, Spearman rho correlation, Independent $\mathrm{T}$ test, Mann-Whitney U-Test, One way ANOVA test (Analysis of variances) and Kruskal-Wallis H-Test were used to present results.

\section{RESULTS}

\section{Regarding demographic characteristics of the patients:}

Ninety seven percent of participants were females and only (3\%) were males. Most of them (82\%) aged more than 40 year old, while (18\%) were less than 40 year old. $(97 \%)$ of patients were married, while only $(3 \%)$ of them were singles. (30\%) of patients' educational level were the primary school only, (29\%) of them were secondary school and only (24\%) were university graduates. Also, (13\%) of them were illiterates and $(4 \%)$ of them studied at khalwa.

Seventy five percent of participants were diagnosed with rheumatoid arthritis, while $(13 \%)$ of them diagnosed with SLE. Also, (4\%) of them diagnosed with connective tissue disease, (2\%) diagnosed with sclera derma and $(6 \%)$ were unknown (Figure-1).

Seventeen percent of patients' source of knowledge about the side effects of CS was health care professionals, $(9 \%)$ of them were family and friends and $(7 \%)$ of them were web sites and media (Figure-2).

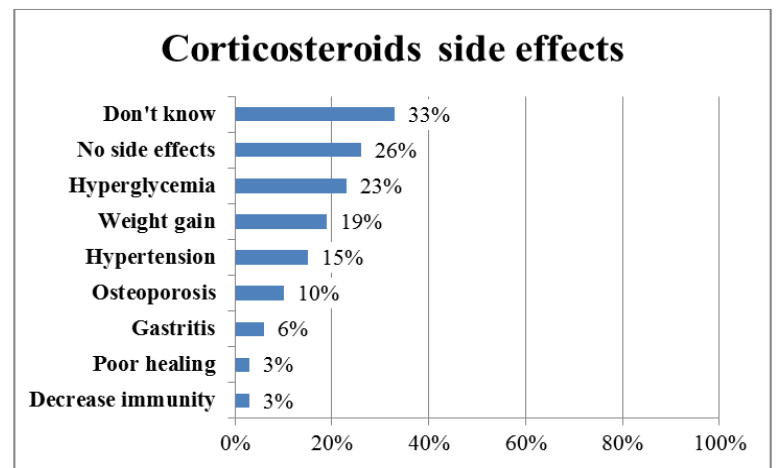

Fig-1: Distribution of corticosteroids side effects knowledge

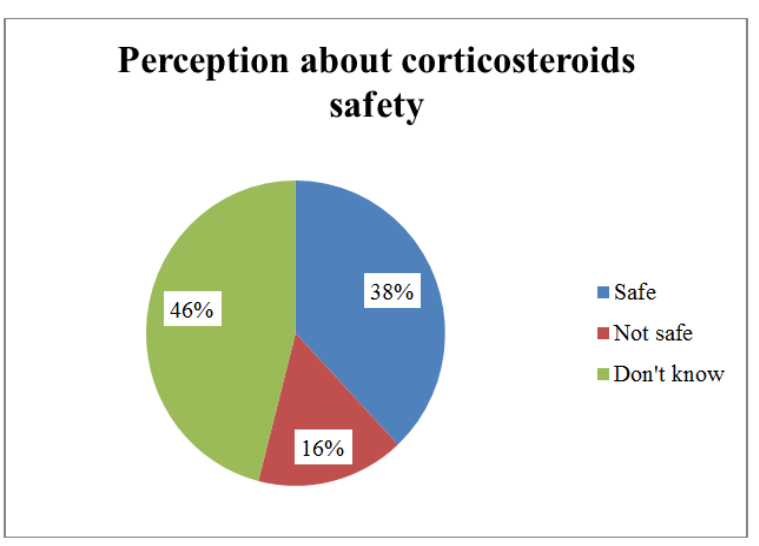

Fig-2: Perception about corticosteroids safety 
Waleed Azhary Sir Alkhatim et al., Sch Acad J Pharm, Mar, 2021; 10(3): 49-53

\section{Drug interactions}

Sixty three percent of patients didn't know about corticosteroids drug interactions. Twenty six percents of patients said that corticosteroids have drug interactions, while $(11 \%)$ of them said that corticosteroids didn't have any interactions (Figure-3).

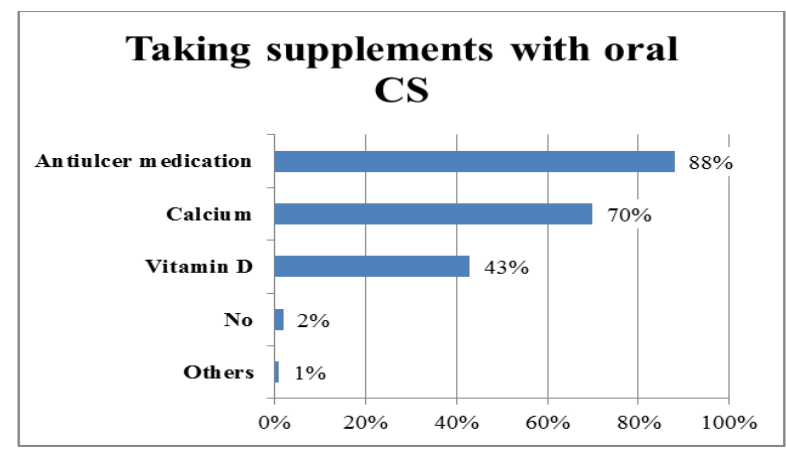

Fig-3: Supplements' taking with oral corticosteroids

\section{Monitoring}

Sixty three percents of patients follow proper morning dosing regimen, while $(37 \%)$ of them do not. Ninety seven percents of patients apply gradual stopping of corticosteroids, while only (3\%) of them apply sudden stopping mood (Figure-4).

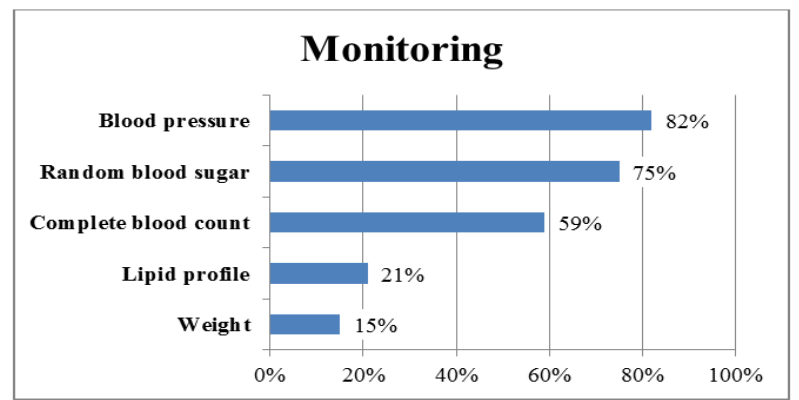

Fig-4: Patients' regular Monitoring

\section{Drug dosing schedule compliance:}

Sixty three percent of patients follow proper morning dosing regimen, while $(37 \%)$ of them do not.

\section{Mood of stopping oral CS}

Seven percent of patients apply gradual stopping of corticosteroids, while only (3\%) of them apply sudden stopping mood. Fifty three percent of patients had a good awareness score while (47\%) of them had a poor one.

The association between demographic data and awareness score recording to the analysis showed that: Females have a better awareness score (53\%) than males with (33\%) awareness score (Figure-5). Patients aged 51-60 have higher awareness score than others.

There was a weak positive relation between the educational level of the patients and the awareness score, i.e. directly proportional, when the educational level increase, the awareness score is also increased (Table-1).

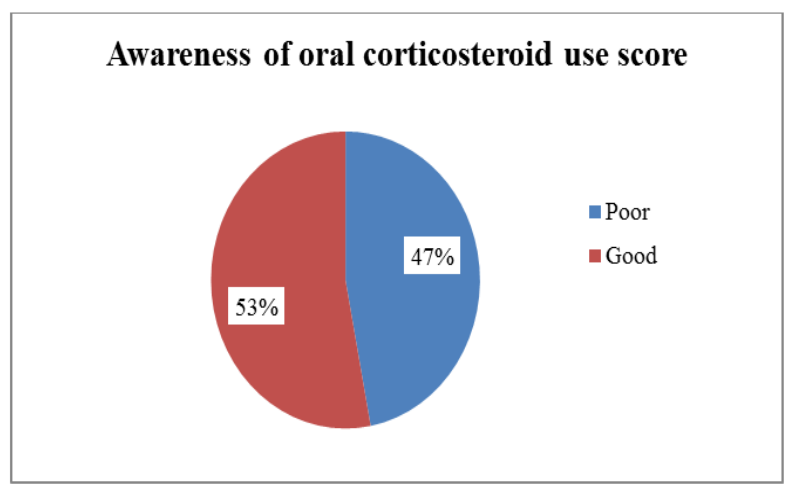

Fig-5: Awareness of oral corticosteroids uses score

Table-1: Correlation between awareness score and educational level

\begin{tabular}{|l|l|l|}
\hline \multicolumn{2}{|l|}{ Spearman's rho correlations } \\
\hline Variables & Awareness of oral corticosteroid use score \\
\hline Educational level & Correlation Coefficient & $0.245^{*}$ \\
\cline { 2 - 3 } & Sig. (2-tailed) & 0.014 \\
\cline { 2 - 3 } & Number & 100 \\
\cline { 2 - 3 } & Strength & Weak \\
\cline { 2 - 3 } & Direction & Positive \\
\hline Age groups & Correlation Coefficient & 0.067 \\
\cline { 2 - 3 } & Sig. (2-tailed) & 0.51 \\
\cline { 2 - 3 } & Number & 100 \\
\cline { 2 - 3 } & Strength & Weak \\
\cline { 2 - 3 } & Direction & Positive \\
\hline \multirow{2}{*}{$*$ Correlation is significant at the 0.05 level (2-tailed). } \\
\hline
\end{tabular}

\section{DISCUSSION}

This study aimed to estimate awareness of rheumatologic disease patient toward oral CS, conducted by questionnaire asking about their knowledge of oral CS side effects, majority of participants answered either did not know or none. Moreover, their perceptions toward oral CS safety were $38 \%, 16 \%$ and $46 \%$ of patient answered safe, not safe and can't tell respectively. In this respect, our study relatively agree with the finding of Amina Mahdy et al 
Waleed Azhary Sir Alkhatim et al., Sch Acad J Pharm, Mar, 2021; 10(3): 49-53

study, which concluded that most patients consider oral CS as highly potentially hazardous drugs, but their knowledge about its associated risks was insufficient.

As observed from the previous results, 37\% and $3 \%$ of patients are non compliant with proper morning dosing regimens or gradual CS tapering off respectively, which posed them to a higher risk of developing adrenal suppression.

In addition, only $88 \%, 70 \%$, and $34 \%$ of patients received concurrent antiulcer medications, calcium or vitamin $\mathrm{D}$, respectively. Ignorance regarding CS risks and necessary precautions highlighted inferior patient education in this regard. Even for widely known serious CS side effects such as bone fractures, osteoporosis, and ulcers that nearly affects $50 \%$ of patients on chronic CS therapy [11], our results and the previous studies have highlighted the lack of concurrent bone loss or ulcer treatment in most patients [12, 13]. The inadequacy of health-care professionals' awareness regarding this matter is probably due to an insufficient consensus between guidelines regarding proper osteoporosis prophylaxis [14]. Nevertheless, most guidelines state that calcium, vitamin D supplementation [13, 15] and prophylactic gastric protectionshould be given routinely to steroid patients.

In this study, $46 \%$ and $63 \%$ of patients can't tell about CS safety and didn't know about their drug interactions, that leads to a lot of side effects might be occurs and patients become vulnerable to complications.

In this study, found the knowledge of patient about oral CS from health-care professional just $17 \%$. In the other hand, $82 \%, 75 \%, 59 \%, 21 \%$ and $15 \%$ of patients were monitor BP, RBS, CBC, lipid profile and weight, respectively. In comparison, information's about CS provided from health-care professional to their patient were relatively lower than practice of them.

Overall awareness score was only 53\% and there is significant relationship (0.029) between educational level and awareness score. Which means prescriber must consider educational level of patient when CS indicated, in order to emphasize more in oral CS use and complications to illiterate patients.

\section{CONCLUSION}

Corticosteroid is a double-edged weapon. While getting maximum benefits and avoiding adverse effects, patients should be aware of adverse effects, precautions and usage of corticosteroids.

This study aimed to assess awareness in patients with rheumatological diseases toward oral corticosteroids includes 100 patients from outpatient clinic of Omdurman military hospital, found their knowledge about steroids and side effects was poor, in contrary the practice of health-care professional in monitoring patients was relatively good. Awareness score was just $53 \%$ (and) directly proportionate to educational level of the patients.

No doubt that continuous improper use of such potent drugs would definitely disrepute such worldwide highly effective drugs.

\section{LIMITATIONS}

The research was the first of its kind since there has been no previous studies conducted in Sudan on patients receiving corticosteroids treatment and also the absence of recorded data base.

The study didn't involve a larger number of participants as there was a lack of patients in the hospital during the period of conducting the research due to the political situation at that time.

\section{What is already known on this topic?}

- No published research about awareness of corticosteroids use among Sudanese population.

- Lack of information regarding adverse effects and monitoring in patient using steroids.

\section{What this study adds}

- Just 53\% good awareness in patient using steroids in Sudan.

- There is significant correlation between awareness of patients using steroids and their educational level.

- The role of healthcare professional in patient's knowledge about steroid is negligent.

\section{Competing Interests: None}

\section{Authors' contributions}

WAS, ZIT, YAA conceived the idea of the study. WAS, YAA, AMB, EMA, SAMI contributed equally to data collection, data analysis. WAS, YAA, AMB, EMA, ZIT, SAMI contributed to manuscript writing and review of the manuscript, all authors' approved the manuscript.

\section{Acknowledgements: None}

\section{REFERENCES}

1. Liu D, Ahmet A, Ward L, Krishnamoorthy P, Mandelcorn ED, Leigh R, Brown JP, Cohen A, Kim H. A practical guide to the monitoring and management of the complications of systemic corticosteroid therapy. Allergy, Asthma \& Clinical Immunology. 2013 Dec;9(1):1-25.

2. Rathi SK, D'Souza P. Rational and ethical use of topical corticosteroids based on safety and efficacy. Indian journal of dermatology. 2012 Jul;57(4):251. 
Waleed Azhary Sir Alkhatim et al., Sch Acad J Pharm, Mar, 2021; 10(3): 49-53

3. Da Silva JA, Jacobs JW, Kirwan JR, Boers M, Saag KG, Inês LB, de Koning EJ, Buttgereit F, Cutolo M, Capell H, Rau R. Safety of low dose glucocorticoid treatment in rheumatoid arthritis: published evidence and prospective trial data. Annals of the rheumatic diseases. 2006 Mar 1;65(3):285-93.

4. Saag KG, Koehnke R, Caldwell JR, Brasington R, Burmeister LF, Zimmerman B, Kohler JA, Furst DE. Low dose long-term corticosteroid therapy in rheumatoid arthritis: an analysis of serious adverse events. The American journal of medicine. 1994 Feb 1;96(2):115-23.

5. Harris Jr ED, Emkey RD, Nichols JE, Newberg A. Low dose prednisone therapy in rheumatoid arthritis: a double blind study. The Journal of rheumatology. 1983 Oct 1;10(5):713-21.

6. Wan ES, Qiu W, Baccarelli A, Carey VJ, Bacherman H, Rennard SI, Agustí A, Anderson WH, Lomas DA, DeMeo DL. Systemic steroid exposure is associated with differential methylation in chronic obstructive pulmonary disease. American journal of respiratory and critical care medicine. 2012 Dec 15;186(12):124855.

7. Liu D, Ahmet A, Ward L, Krishnamoorthy P, Mandelcorn ED, Leigh R, Brown JP, Cohen A, Kim H. A practical guide to the monitoring and management of the complications of systemic corticosteroid therapy. Allergy, Asthma \& Clinical Immunology. 2013 Dec;9(1):1-25.

8. Manson SC, Brown RE, Cerulli A, Vidaurre CF. The cumulative burden of oral corticosteroid side effects and the economic implications of steroid use. Respiratory medicine. 2009 Jul 1;103(7):97594.

9. Shah M, Chaudhari S, McLaughlin TP, Kan HJ, Bechtel B, Dennis GJ, Molta CT. Cumulative burden of oral corticosteroid adverse effects and the economic implications of corticosteroid use in patients with systemic lupus erythematosus. Clinical therapeutics. 2013 Apr 1;35(4):486-97.

10. Aulakh R, Singh S. Strategies for minimizing corticosteroid toxicity: A review. In: Indian Journal of Pediatrics. 2008.

11. Lane NE. An update on glucocorticoid-induced osteoporosis. Rheumatic Disease Clinics of North America. 2001 Feb 1;27(1):235-53.

12. Van Staa TP, Leufkens HG, Abenhaim L, Begaud B, Zhang B, Cooper C. Use of oral corticosteroids in the United Kingdom. Qjm. 2000 Feb 1;93(2):105-11.

13. Peat ID, Healy S, Reid DM, Ralston SH. Steroid induced osteoporosis: an opportunity for prevention?. Annals of the rheumatic diseases. 1995 Jan 1;54(1):66-8.

14. Willenberg HS, Lehnert $H$. Basics and management of glucocorticoid-induced osteoporosis. Internist. 2008;49(10):1186-96.

15. Buckley L, Greenwald M, Hochberg M, Lane NE, Lindsey S, Paget S, Saag K, Simon L. Recommendations for the prevention and treatment of glucocorticoid-induced osteoporosis: 2001 update: American College of Rheumatology Ad Hoc Committee on glucocorticoid-induced osteoporosis. Arthritis and Rheumatism. 2001;44(7):1496-503. 